\title{
Archéopages
}

Archéopages

Archéologie et société

Hors-série 2 | 2010

Archéologie sans frontières

\section{Pour un nouveau programme de recherche à Sanghol}

Jean-Yves Breuil et Marilyne Bovagne

\section{OpenEdition}

1 Journals

Édition électronique

URL : https://journals.openedition.org/archeopages/740

DOI : 10.4000/archeopages.740

ISSN : 2269-9872

Éditeur

INRAP - Institut national de recherches archéologiques préventives

Édition imprimée

Date de publication : 1 octobre 2010

Pagination : 14-19

ISSN : $1622-8545$

Référence électronique

Jean-Yves Breuil et Marilyne Bovagne, "Pour un nouveau programme de recherche à Sanghol ", Archéopages [En ligne], Hors-série 2 | 2010, mis en ligne le 01 octobre 2010, consulté le 23 février 2023. URL : http://journals.openedition.org/archeopages/740 ; DOI : https://doi.org/10.4000/ archeopages. 740 

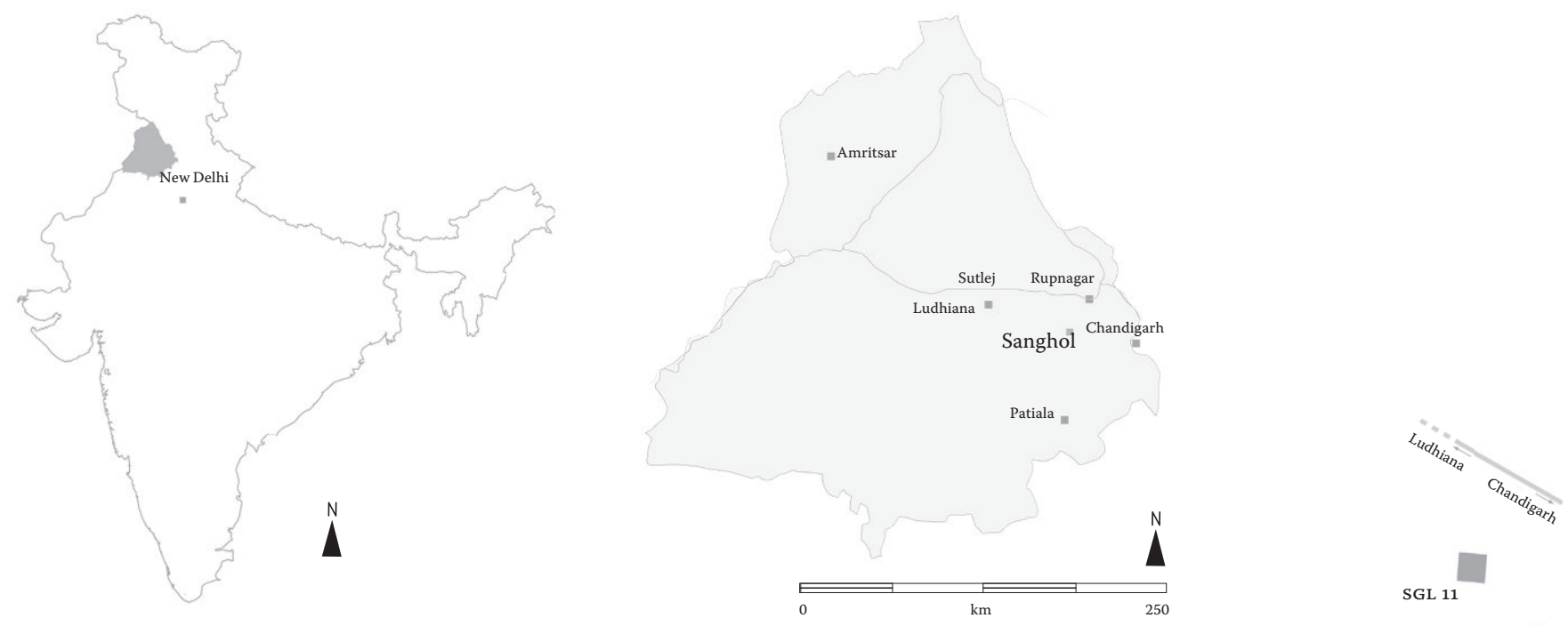

SGL 11

$\mathbf{I}$

[Fig. 1] Le site de Sanghol.

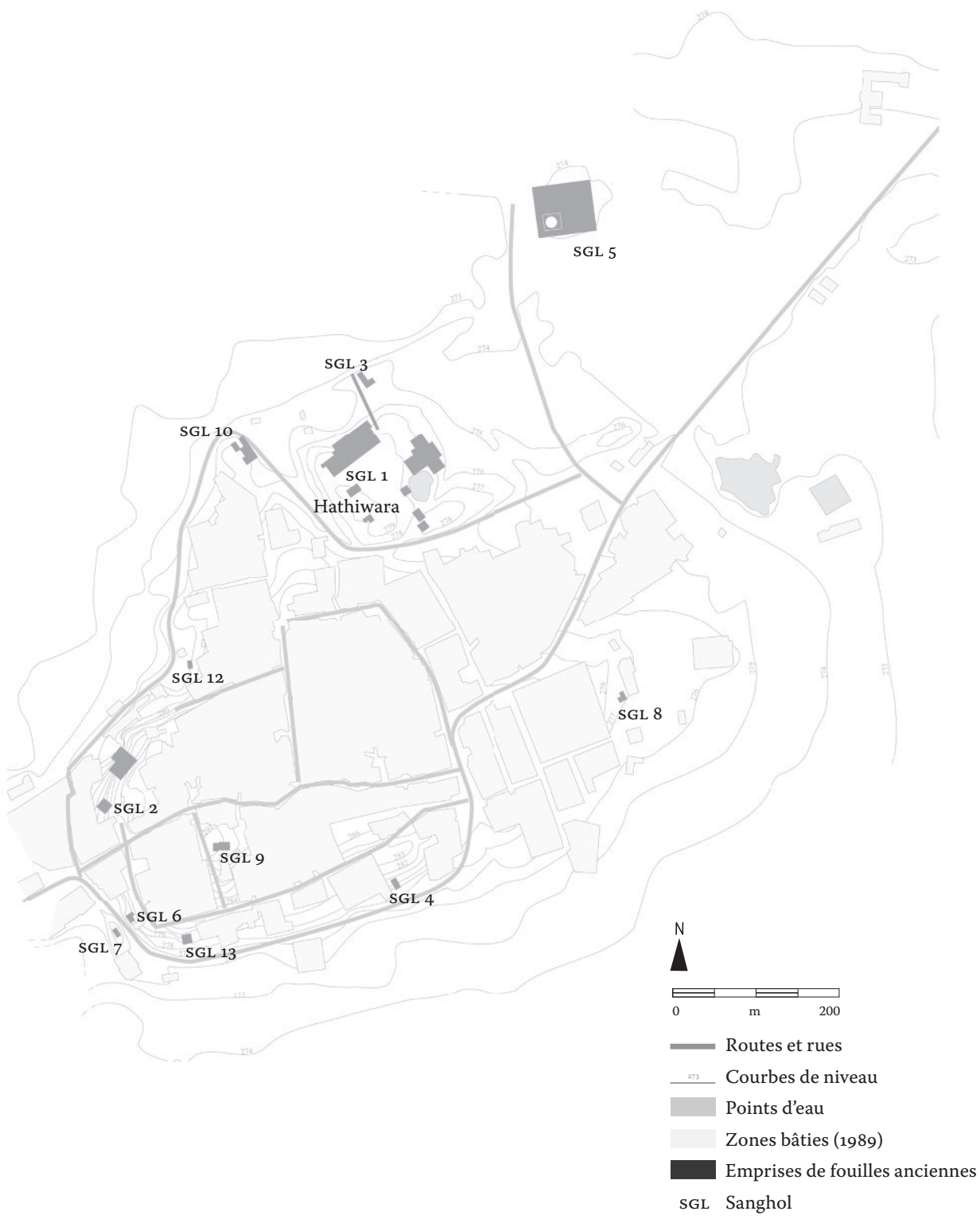


puisqu'elle a permis d'obtenir une complémentarité des différents domaines d'activité en exploitant au mieux les compétences des uns et des autres. Cette recherche a, de plus, largement bénéficié des apports transdisciplinaires en sollicitant des laboratoires ou spécialistes extérieurs pour des études śpécifiques. Enfin, la confiance mutuelle entre les différents acteurs a été un point important qui a contribué au succès de ces missions et a ainsi permis de répondre au mieux à la problématique de début. Les différentes interventions menées depuis 2005 ont donc permis de mettre en évidence le caractère simultané du dépôt des corps lors de plusieurs crises de mortalité successives. Ces premiers résultats permettent d'envisager que ce secteur fut le lieu de regroupement des victimes de plusieurs épidémies. De la même façon, loriginalité des pratiques funéraires est reconnue et reste désormais à interpréter. L'objectif des années à venir est de mieux cerner la nature des décès (peste antonine de la seconde moitié du $\mathrm{II}^{\mathrm{e}}$ siècle ?) et le recrutement des défunts (population étrangère à Rome, famille des equites singulares Augusti ?).

Blanchard Ph., 2006, La Gestion des cadavres en temps de crise, Exemples archéologiques médiévaux et modernes dans l'Europe de l'Ouest, Mémoire de master, Université de Tours, 2 vol., 114 p.

Castex D., Blanchard Ph., Giuliani R., Ricciardi M., 2007, «Les ensembles funéraires du secteur central de la catacombe des saints Pierre-et-Marcellin (Rome, $\mathrm{I}^{\mathrm{er}}-\mathrm{III}^{\mathrm{e}} \mathrm{s}$.) : caractérisation, hypothèses d'interprétations et perspectives de recherches ", Mélanges de l'École française de Rome, 119-1, p 274-282.

Castex D., Blanchard Ph., Reveillas H., Kacki S., Giuliani R. 2009 «Les sépultures du secteur central de la catacombe des saints Pierreet-Marcellin (Rome). État des analyses bio-archéologiques et perspectives ", Mélanges de l'École française de Rome, 121-1, p. 287-297.

Devièse T., Vanhove C., Chapoulie R., Blanchard Ph, Colombini M.-P., Regert M., Castex D., Sous Presse « Détermination et fonction des substances organiques et des matières minérales exploitées dan les rites funéraires de la catacombe des saints Pierre-et-Marcellin à Rome ( $\mathrm{e}^{\mathrm{er}}$-III ${ }^{\mathrm{e}}$ siècles) , in CARTron I., CASTEX D., Georges P., Charegeat M., Vivas M., De corps en corps : traitement et devenir du cadavre, Actes des séminaires de la Maison des sciences de l'homme d'Aquitaine 2007-2008, MSHA Éditions.

GIULANI R., novembre-décembre 2008, « Ad duas lauros », Dossiers de l'Archéologie, $\mathrm{n}^{\circ}$ 30, p. 48-55.

GiUliani R., CASTEX D., 2006-2007, « La scoperta di un nuovo santuario nella catacomba dei SS. Marcellino e Pietro e lo scavo antropologico degli insiemi funerari annessi. Risultati preliminar di un'indagine multidisciplinare ", in Rendiconti della Pontificia Accademia Romana di Archeologia, 79, p. 83-124.

Guyon J. , 1987, Le Cimetière « aux deux lauriers », Recherches sur les catacombes romaines, Rome, Pontificio istituto di archeologia christiana /École française de Rome.

Souquet-Leroy I., Blanchard Ph., Castex D., Poulle P., 2007, «Stratégie de fouille d'un ensemble de sépultures multiples à Issoudun ", Archéopages 19, Inrap, p. 60-69.

\section{Pour un nouveau programme de recherche à Sanghol}

\author{
Jean-Yves Breuil \\ Marilyne Bovagne \\ Inrap
}

$\mathrm{S}$ anghol, au Penjab, est l'un des sites archéologiques principaux d'Inde nordoccidentale. Il est situé dans une région de plaine, intermédiaire entre le Gandhara et Mathura, à proximité de la rivière Sutlej et des anciennes voies de communication entre le Nord-Ouest et les régions du Sud

(dakshināpatha, la route du Sud) et entre le Nord-

Ouest et Pataliputra à l'est (uttarāpatha, la route du Nord). Il est occupé depuis 4000 ans, s'étend sur près de 75 hectares et atteint, à son point culminant, 18 mètres d'épaisseur stratigraphique. C'est un site d'importance majeure en Inde du Nord, tant pour la durée de son occupation que pour son patrimoine bouddhique : il est renommé notamment pour ses stūpas et sculptures du début de l'ère chrétienne.

Les nombreuses fouilles conduites par le Punjab Department of Archaeology et l'Archaeological Survey of India ont donné lieu à un nombre extrêmement restreint de publications. De fait, bien que suscitant maintes attentes dans la communauté scientifique, en particulier en tant que référence régionale, l'histoire du site-phare du Penjab demeure très méconnue.

Himanshu P. Ray, professeur d'histoire à la Jawaharlal Nehru University de New Delhi, initiant un nouveau projet de recherche sur Sanghol, a sollicité une équipe de spécialistes (M. Bovagne, J.-Y. Breuil et S. Gill, historienne de l'art indien) pour une première mission d'expertise de deux semaines qui s'est déroulée en mars 2010. L'objectif de cette investigation préliminaire était de soumettre des propositions contribuant à définir le futur programme de recherche archéologique.

Situation. Sanghol se situe à $250 \mathrm{~km}$ au nordnord-ouest de New Delhi, sur un axe majeur reliant deux grandes villes du Penjab, Ludhiana (à $54 \mathrm{~km}$ ) et Chandigarh (à 40 km) [Fig.1]. Localement connu sous le toponyme «Ucha Pind», le village, à vocation agricole et commerciale, compte environ 7 ooo habitants. Il s'étend majoritairement sur un monticule d'origine anthropique [Fig.2] et, hormis une réserve archéologique au nord-est du village, dans laquelle se situe une partie des fouilles anciennes, la colline et ses pentes sont complètement urbanisées. Les autres parcelles environnantes sont dévolues à l'agriculture. L'expansion du village se fait parfois au détriment des vestiges archéologiques sous-jacents. Un exemple parmi d'autres : des vestiges, récemment mis au jour lors des travaux d'agrandissement d'une école (Gvt High School), ont rapidement été escamotés. Les villageois, craignant souvent (à tort) l'expropriation à cause de la question archéologique, préfèrent taire les découvertes fortuites.

La topographie actuelle traduit l'existence de la cité ancienne. Le tell, qui culmine à $291 \mathrm{~m}$ d'altitude, est haut d'une vingtaine de mètres; il mesure 500 m d'est en ouest et $400 \mathrm{~m}$ du nord au sud. Il présente des parois abruptes sur trois de ses côtés et un pendage plus léger vers l'est. À l'intérieur du village, certaines rues circulaires pérennisent probablement une structuration plus ancienne.

Les vestiges archéologiques connus ne se limitent pas au tell. Les 13 campagnes réalisées entre 1969 et 1990, qui ont généré 13 secteurs de fouilles différents, ont permis de mettre en évidence l'étendue du site sur au moins $1400 \mathrm{~m}$ d'est («Sanghol 2 ») en ouest (« Sanghol $11 »)$, 


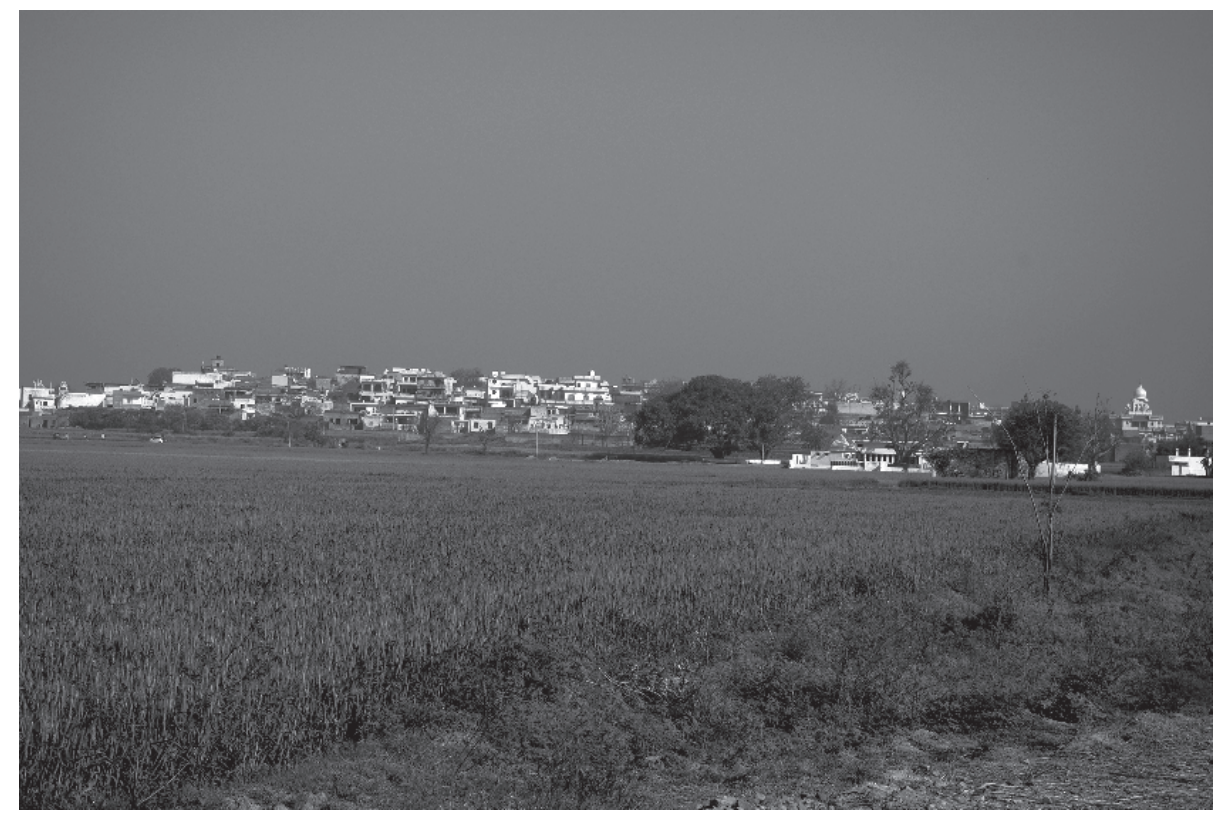

9

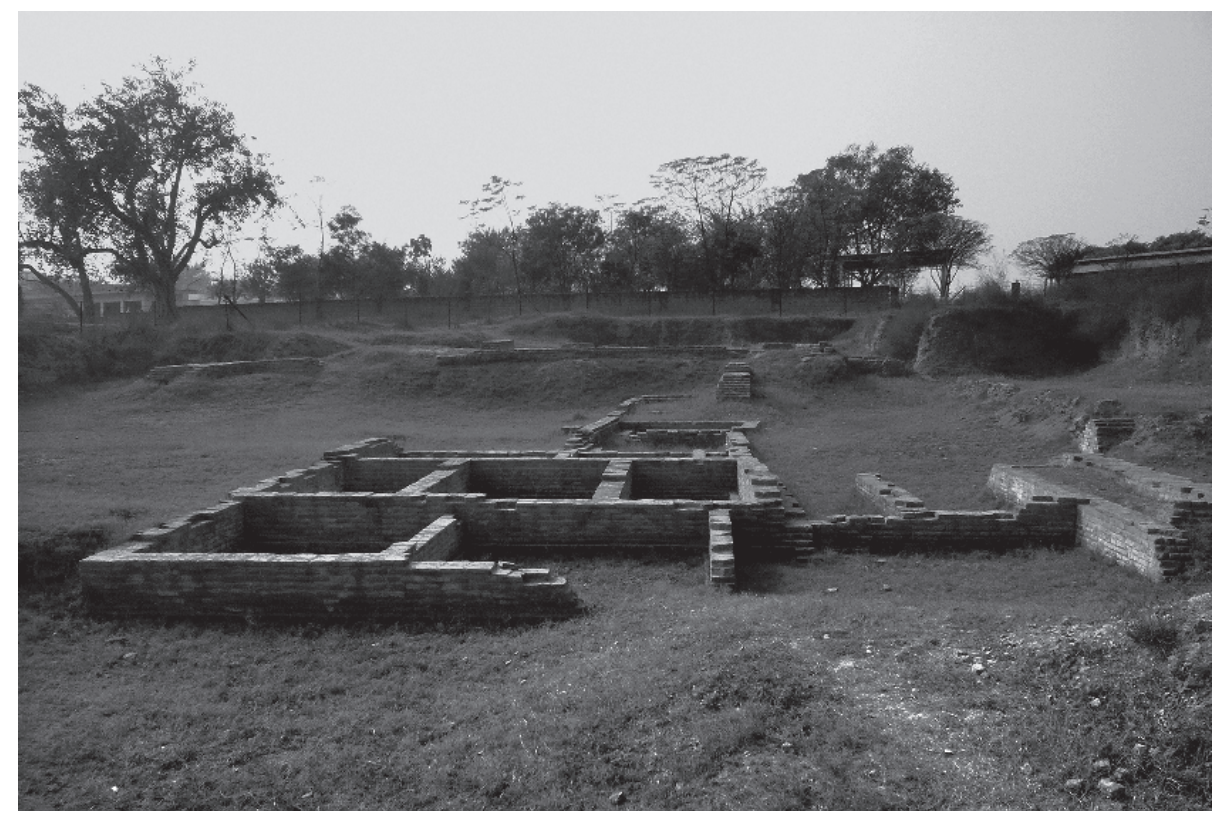

[Fig.2] Vue générale du village installé sur le tell.
[Fig.3] Vestiges conservés sur le site du complexe d'Hathiwara.
[Fig.4] Un élément sculpté de la balustrade du stupa principal.

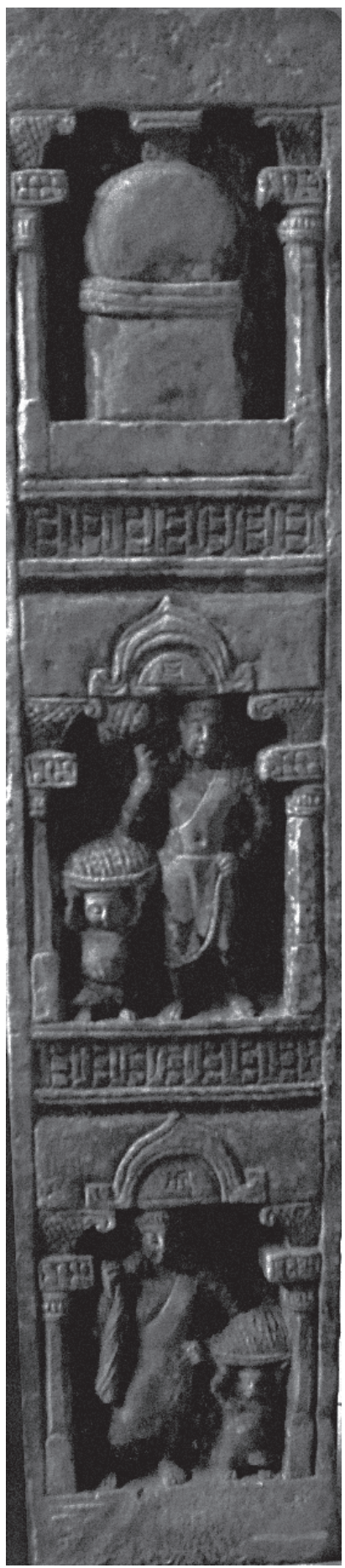


et $500 \mathrm{~m} \mathrm{du}$ nord ( $«$ Sanghol $10 »)$ au sud («Sanghol 4 »). Les données issues de ces fouilles n'ont fait l'objet d'aucune publication importante. Cependant il est possible, à partir des comptes rendus succincts publiés annuellement dans Indian Archaeology A Review (IAR) d'esquisser les grands traits de la séquence stratigraphique et de l'organisation spatiale du site.

Chronologie. Globalement, les fouilles anciennes ont mis en évidence huit grandes phases inégalement représentées dans les 13 zones investies (Michon, 2010). L'occupation du site s'étend, de manière continue, de la période harappéenne tardive, vers 1900 avant l'ère chrétienne, jusqu'à l'époque de l'empire Gupta (320-600). L'ensemble des trouvailles attestent clairement la succession des diverses cultures matérielles jusqu'au vi ${ }^{\mathrm{e}}$ siècle de notre ère : Harappa, Bara, Painted Gray Ware, Black Slipped/ Northern Black Polished Ware, Indo-Parthe, Kouchane et Gupta. Ensuite, quelques traces des invasions des Indo-Hephthalites (Huns) au $\mathrm{VI}^{\mathrm{e}}$ siècle, puis des campagnes musulmanes à la suite de la conquête du Sind (à partir du VIII $^{\mathrm{e}}$ siècle) semblent avoir été identifiées dans certaines zones du site. Après un possible hiatus de 200 ans, l'occupation reprend vers 1200 pour se poursuivre jusqu'à nos jours.

Structuration spatiale. La distribution des vestiges au gré des différents secteurs de fouille permet d'ébaucher une première organisation spatiale générale du site. Les cultures « protohistoriques » (de Harappa à la NBP Ware) caractérisées par une architecture de brique crue semblent circonscrites au monticule principal. Cependant, aucun plan d'ensemble des structures n'a encore été esquissé.

La phase kouchane $\left(\mathrm{I}^{\mathrm{er}}-\mathrm{II}^{\mathrm{e}}\right.$ siècles de notre ère) est la mieux identifiée dans le paysage de Sanghol. Sur le sommet du tell protohistorique, plusieurs mètres de stratigraphie attestent de nombreux états architecturaux relatifs à de l'habitat faisant usage de la brique cuite. La partie basse, au nordest, est aussi investie ; au lieu-dit Hathiwara [Fig.3] est ainsi érigé un ensemble bâti dit « palatial » comportant un mur de fortification quadrangulaire, des bâtiments administratifs et des habitations. Un rempart de terre a également été mis en évidence dans ce secteur. Il a pu endiguer les crues de la Sutlej dont le parcours ancien, qui se devine à l'examen de la topographie actuelle, bordait vraisemblablement Sanghol. Cet affluent de l'Indus, dont le cours actuel passe à près de $15 \mathrm{~km}$ plus au nord, est l'un des éléments constitutifs importants du paysage ancien de Sanghol. À environ $250 \mathrm{~m}$ au nord-est (Sanghol 5), un établissement bouddhique de la période kouchane, comprenant un stūpa principal et d'autres aménagements (petits stupas, monastère), a été mis au jour. Cent dix-sept éléments de la balustrade sculptée du stupa principal ont été découverts soigneusement rangés dans une fosse à proximité [Fig.4]. Cette balustrade en grès rose tacheté, représentant des déesses féminines, est caractéristique des productions des ateliers kouchans de Mathura, situé $400 \mathrm{~km}$ plus au sud (Gill, 2010). Elle illustre l'importance de Sanghol dans le paysage politique et religieux de l'Inde du Nord. L'implantation inhabituelle de cet établissement bouddhique en très proche périphérie d'un ensemble urbain est une autre problématique importante de la recherche sur Sanghol. Un autre ensemble monacal contemporain du stupas, découvert $450 \mathrm{~m}$ encore plus au nord-est, sur un terrain a priori dépourvu de vestiges antérieurs, atteste l'extension de l'occupation de Sanghol au $\mathrm{II}^{\mathrm{e}}$ siècle de notre ère. Enfin, des témoignages plus discrets datés des phases Gupta et médiévales ont été identifiés sur le tell, et aussi en contrebas, au sudest du village (Sanghol 8) montrant la permanence de l'occupation sur une superficie importante.

Constats de la mission. Cette description très succincte de l'histoire de Sanghol laisse évidemment en suspens de nombreuses questions et souffre de l'absence cruelle d'une cartographie et d'une synthèse reliant entre elles les séquences stratigraphiques et les données des 13 zones de fouille. Aucune étude n'a exploité les données archéologiques dans leur globalité, ni rendu compte de Sanghol dans son intégralité, tant diachronique que topographique. Seule une partie des sculptures mises au jour en 1984 a été rapidement publiée (Gupta, 1985) ainsi qu'un ensemble de monnaies (Sharma, 1986). Au-delà des notices de fouilles parues annuellement, mais dépourvues de plan et de stratigraphie (IAR), il existe aussi deux courts articles concernant les campagnes des années 1986 et 1987 (Margabandhu, Gaur, 1987 et 1988). Le volume paru en 2010 sous la direction de H. P. Ray (Ray, 2010) recueille une douzaine d'articles divers (histoire, histoire de l'art et archéologie) sur la documentation de fouille, l'histoire du Penjab ou encore les sculptures kouchanes. Inaugurant en quelque sorte le nouveau programme de recherche archéologique, cette édition pourrait être le premier élément initiant une véritable politique de publication des données sur et autour de Sanghol.

Un examen préliminaire, mais détaillé, de la documentation de fouille montre qu'elle est actuellement partielle, endommagée et éparpillée en trois lieux (au Department of Archaeology à Chandigarh ${ }^{\mathbf{1}}$, au Purana Qila Museum à Delhi, et à Qila Mubarak à Patiala).

Les collections archéologiques sont en partie conservées au Musée archéologique de Sanghol, notamment la majorité des sculptures pour lesquelles il a été construit, mais également dans les trois autres lieux mentionnés pour la documentation. De manière générale, l'état de conservation et l'accessibilité de la documentation archéologique (documents et objets) sont problématiques.

Deux visites du site ont permis d'aborder la morphologie et la structuration générale de l'occupation humaine et de localiser les différents secteurs de fouille dans la perspective d'une future cartographie et d'une évaluation des zones 
en danger, des secteurs à investir prioritairement, des secteurs à fort potentiel stratigraphique, etc.

Grâce à la mémoire d'anciens fouilleurs, il a été possible d'identifier, parfois difficilement, l'emplacement exact de chacune des treize emprises de fouille [Fig.1]. Trois zones, le « complexe palatial » d'Hathiwara (Sanghol 1), l'ensemble religieux du grand stupas (Sanghol 5) et le monastère plus éloigné (Sanghol 11), font l'objet d'un « classement » par l'Archaeological Survey of India, autorité compétente en matière de fouille et de conservation, et constituent des parcs archéologiques clos dans lesquels de futures fouilles sont inenvisageables. Dans ces trois parcs, les vestiges, apparemment ceux visibles dans les derniers temps de la fouille, ont été restaurés/ restitués ; il s'agit d'architecture en brique cuite appartenant à l'occupation kouchane [Fig.3]. Les autres zones de fouilles ont été soit remblayées pour l'aménagement du village, soit laissées ouvertes depuis plus d'une vingtaine d'années à la grâce des troupeaux de chèvres, comblées progressivement par l'érosion des bermes et les détritus. Il n'y a pas de surveillance archéologique mise en place lors des travaux d'aménagement effectués dans le village.

Propositions. Nous avons fait le choix d'articuler notre contribution au futur programme de recherche archéologique autour des points suivants : réinvestir, sauvegarder, analyser et diffuser les recherches anciennes; optimiser les nouvelles interventions archéologiques; intégrer une dimension pluridisciplinaire et multi-partenariale dans le nouveau projet; développer une politique de protection et de valorisation du site. Ces aspects ont été développés lors d'un colloque/table ronde «Sanghol and the archaeology of Punjab » qui s'est tenu à New Delhi les 15 et 16 mars 2010, à travers une communication intitulée «A few proposals for a future archaeological research on Sanghol site» (Bovagne, Breuil, Gill). L'objectif est une compréhension « holistique » du site en termes de chronologie, de référentiel culturel, de restitution paysagère et d'insertion dans l'histoire régionale.

Il paraît d'abord urgent d'entreprendre les deux actions suivantes : rassembler, numériser et synthétiser les données anciennes et mettre en œuvre un relevé cartographique général du site.

Les différentes fouilles se répartissent en effet de manière assez homogène au sein du village et de ses faubourgs cultivés. L'analyse synthétique de l'ensemble des données pourra alors permettre de restituer la topographie ancienne du site. Ce travail passe en premier lieu par la stabilisation et l'inventaire de la documentation de fouille. Son exploitation s'avère assez complexe. Partielle, inégalement conservée d'un carré de fouille à l'autre, inexistante pour certains secteurs, il paraît nécessaire de l'exploiter suffisamment, sans pour autant investir trop de temps et d'énergie pour un résultat encore incertain. Par ailleurs, numériser et inventorier les données permettent de les sauvegarder de manière plus sûre et de les rendre accessibles à un plus grand nombre. Cette démarche doit s'accompagner

de la mise en place d'une base de données relationnelle commune aux différents chercheurs.

Une seconde action prioritaire est d'établir une cartographie complète et détaillée du site. Cette cartographie est l'outil indispensable pour traiter les données anciennes (géo localisation des données), comprendre dans le détail la topographie du site, établir un plan des secteurs menacés et déterminer les futures zones de fouille.

Un troisième temps serait, à l'aune des deux actions précédentes, de pratiquer des tranchées d'évaluation ciblées, en fonction du plan d'urbanisation. L'intervention archéologique doit forcément intégrer une dimension pluridisciplinaire, notamment géomorphologique, afin de comprendre l'évolution du site dans son environnement naturel.

D'autre part, il est important de mettre en place au sein même du village un travail de préservation et de valorisation des vestiges. La sensibilisation des villageois au patrimoine archéologique pourrait passer par la mise en place d'une signalisation des vestiges (quasi inexistante), par des actions de valorisation à travers le musée de Sanghol, et par la formation de guides et gardiens locaux. Il s'agit d'améliorer la connaissance du site et de favoriser la collaboration des habitants et des instances locales.

Le nouveau projet de recherche doit fédérer les différents acteurs de l'archéologie concernés par Sanghol et les impliquer dans une démarche commune de publication des données et de préservation patrimoniale. L'intervention de diverses institutions indiennes sur le site de Sanghol a contribué à fractionner et éparpiller l'information archéologique, mais leur intérêt renouvelé dans ce projet témoigne de sa valeur et peut être un gage de réussite, même si cela complexifie le processus de décision et de mise en œuvre. Force est de constater l'absence étonnante, au sein des organismes indiens (peut-être due en partie à la régression du nombre de fouilles ces dernières années), d'une nouvelle génération d'archéologues susceptibles de gérer ce type d'intervention et de fédérer des intérêts institutionnels distincts. L'intervention sur ce genre de site relève, d'une certaine façon, davantage des méthodes de l'archéologie préventive que des habitudes de l'archéologie programmée : optimisation des interventions

2 Cette table ronde
réunissait d'anciens
acteurs de l'archéologie
à Sanghol, dont plusieurs
archéologues de
l'Archaeological Survey
of India et du Department
of Archaeology of Punjab
ayant fouillé le site de
Sanghol dans les années
1970 et 1980, et des
chercheurs en archéométrie,
cartographie, histoire
de l'art et archéologie,
potentiellement impliqués
dans le nouveau projet
de recherche. La table
ronde a permis d'aborder
également d'autres sites
archéologiques du Penjab

et l'histoire récente de cet État. 3 Lors de la communication en appui de cet aspect, a été présenté un poste traitant des différentes échelles d'études et possibilités liées à un système d'information géographique (SIG) Ce poster a été réalisé par Séverine Sanz, archéologue au CNRS (UMR 5140 de Lattes) et membre du réseau IsA. 
sur une courte durée, accommodation de la recherche et des aménagements et travail en multi partenariat. C'est l'ensemble de ce défi qu'une collaboration extérieure à l'administration indienne, celle des archéologues de l'Inrap par exemple, pourrait relever.

GILL S., 2010, "Celestial women in a ring around the Buddhist stūpa: the case of Sanghol », in RAY H.P. (ÉD.), Sanghol and the Archaeology of Punjab, New Delhi, p. 129-151.

Gupta S. P. (ÉD.), 1985, Kushana sculptures from Sanghol $\left(1^{s t}-2^{\text {nd }} A D\right)$. A recent discovery, National Museum, New Delhi.

Indian Archaeology - A Review, «Exploration and Excavations: excavation at Sanghol, district Ludhiana », 1968-69, 1969-70, 1970-71, 1971-72, 1972-73, 1977-78, 1980-81,1984-85, 1985-86, 1986-87, 1987-88, 1988-89, 1989-90.

Margabandhu C., Gaur G. S., 1987, « Some fresh evidence from Sanghol excavations, $1986 »$, Purāttna, n 16 , New Delhi, p. 74-78

Margabandhu C., Gaur G. S., 1988, «Sanghol excavations 1987: some new evidences ", Purātattva - Bulletin of the Indian Archaeological Society, ${ }^{\circ}$ 17, New Delhi, p. 1-5.

Michon D., 2010, « The Excavation at Sanghol in Context », in Ray H. P. (ÉD.) Sanghol and the Archaeology of Punjab, New Delhi, p. 69-106.

Ray H.P.(ÉD.), 2010, Sanghol and the Archaeology of Punjab, New Delhi. Sharma G. B., 1986, Coins, seals and sealings from Sanghol, Department of Cultural Affairs, Archaeology and Museums, Punjab.

\section{Paharpur, ou comment protéger et restaurer un site dégradé grâce à l'expertise archéologique}

\author{
Jean-Yves Breuil \\ Inrap \\ Sandrine Gill \\ Creops
}

$\mathrm{L}$ e monastère bouddhique de Paharpur, aujourd'hui le Bangladesh, a été fondé à la fin du viII ${ }^{\mathrm{e}}$ siècle. Centre intellectuel de renom du bouddhisme d'Inde orientale d'époque Pāla, considéré à son apogée comme le plus grand monastère bouddhique du sous-continent indien, il possède en son centre un imposant monument de culte cruciforme, dont le modèle fut diffusé dans toute l'Asie du Sud-Est, et un ensemble exceptionnel de 2800 plaques de terre cuite figurées, disposées en frise sur les différentes terrasses du monument. Ces principaux critères lui doivent de figurer depuis 1985 sur la liste du Patrimoine mondial de l'Unesco. Cette mise en lumière internationale s'est malheureusement accompagnée d'une campagne de restauration tout à fait excessive qui a fortement dégradé l'authenticité d'un site déjà fragilisé par des décennies d'exposition à des contraintes environnementales fortes et à un vandalisme récurrent. Plusieurs missions initiées par le Centre du Patrimoine mondial de l'Unesco ont tenté de freiner ce processus et d'améliorer la gestion archéologique de ce site bouddhique très réputé.

Un grand monastère Pāla. Le site de Paharpur se situe près du village de Jaipurhat dans l'actuel district de Naogaon, dans le nord-ouest du Bangladesh, à 80 km au nord-est de Rajshahi et des rives du Gange [Fig.1]. Il abrite les vestiges d'un immense monastère bouddhique, dont la taille exceptionnelle le distingue de la nébuleuse des sites bouddhiques actifs à l'époque Pāla (VIII ${ }^{\mathrm{e}}-\mathrm{XII}^{\mathrm{e}}$ siècles). Au centre du monastère trône un monument de culte imposant [Fig.2], interprété comme une combinaison entre un temple et un stupa, monument bouddhique par excellence.

Paharpur, l'ancienne Somapura Mahāvihāra, fut fondé par le second empereur de la dynastie indienne Pāla, Dharmapāla, qui régna entre 770 et 810. À cette époque, la région de Paharpur, le Varendra, comme toute l'Inde orientale, était sous la domination de la dynastie Pāla dont les dirigeants étaient de fervents défenseurs du bouddhisme, sans qu'ils en aient fait pour autant une religion d'État. Ils parrainèrent la construction de nombreux stupas et vihara (monastères), centres d'apprentissage du bouddhisme du Grand Véhicule (Mahāyāna). De grands sites bouddhiques connurent alors un développement important pendant plusieurs siècles, tels Mainamati au sud-est du Bangladesh ou Nalanda au Bihar indien. $\mathrm{Au} \mathrm{XII}{ }^{\mathrm{e}}$ siècle, un moine du monastère de Nalanda, en pérégrination au nord du Bengale, décrit Paharpur comme « un festin exceptionnel pour les yeux du monde ». La renommée du monastère de Paharpur est attestée dans les sources indiennes, tibétaines et chinoises. Ce site, qui apparaît de nos jours isolé dans la campagne bangladaise, était à l'origine entouré par un important réseau de sites bouddhiques, comme en témoignent la présence, dans un rayon de $40 \mathrm{~km}$, de plusieurs monastères récemment redécouverts : Halud vihāra, Jagdal vihāra, Jagjivanpur, Vasu Bihar et Bihar (Breuil, Gill, 2007). Laissé à l'abandon au moment de la conquête islamique au début du XIII ${ }^{\mathrm{e}}$ siècle, le site de Paharpur fut redécouvert au début du XIX ${ }^{\mathrm{e}}$ siècle par le Britannique Buchanan Hamilton. Les premières fouilles commencèrent en 1923 sous l'égide de l'Archaeological Survey of India et se déroulèrent pendant une dizaine d'années jusqu'en 1934, principalement sous la direction de K. N. Dikshit (Dikshit, 1938). Les fouilles furent accompagnées d'une campagne de restauration de certaines parties du monument. À la suite de la demande du gouvernement du Bangladesh, Paharpur fut classé sur la liste du Patrimoine mondial culturel en 1985, en même temps que la mosquée de Bagerhat (Khalifatabad) datée $\mathrm{du} \mathrm{Xv}^{\mathrm{e}}$ siècle et située dans le sud-ouest du pays.2. Depuis les années 1980, des fouilles de petite superficie sont irrégulièrement menées, par le Département d'Archéologie du Bangladesh, dans la cour et les cellules du monastère.

Le site se compose d'un monastère principal construit en briques, de plan carré (environ 280 m de côté), composé de 177 cellules de moine ouvrant sur une cour au centre de laquelle est érigé le monument de culte cruciforme (dont la partie préservée mesure encore $22 \mathrm{~m}$ de hauteur). Un certain nombre de structures annexes sont bâties dans la cour et à l'extérieur du monastère [Fig.1].

Les investigations récentes ont confirmé l'existence de quatre états de construction et reconstruction, tant pour le monastère principal 